\title{
ON THE NORMAL STABILITY OF FUNCTIONAL EQUATIONS
}

\author{
ZENON MOSZNER
}

\begin{abstract}
In the paper two types of stability and of b-stability of functional equations are distinguished.
\end{abstract}

\section{The translation equation}

It is possible to distinguish two types of the stability of functional equations and the same is possible for the b-stability. We will show this difference for the translation equation and for the dynamical system. The equation

$$
F(F(\alpha, x), y)=F(\alpha, x y),
$$

where $F: S \times G \rightarrow S, S$ is an arbitrary metric space with the metric designed $|a-b|$ (not necessary the norm) and $G$ is a groupoid with the operation ".", is said to be the translation equation, which has many applications [13].

Definition 1. Equation (1) is said to be stable in the Ulam-Hyers sense (in short "stable") if for every $\epsilon>0$ there exists $\delta>0$ such that for every

Received: 28.09.2015. Revised: 24.12.2015. Accepted: 07.01.2016.

(2010) Mathematics Subject Classification: 39B82, 39B62.

Key words and phrases: Stability, normal stability, b-stability, uniform b-stability, normal uniform b-stability, restricted uniform b-stability, translation equation, exponential Cauchy equation, Dhombres' equation, sine, cosine and quadratic equations, squares of Cauchy and Jensen equations, Lobachevski's equation, Pexider's equation. 
function $H: S \times G \rightarrow S$ such that

$$
|H(H(\alpha, x), y)-H(\alpha, x y)| \leq \delta \quad \text { for } \alpha \in S \text { and } x, y \in G
$$

there exists the solution $F$ of (1) such that

$$
|H(\alpha, x)-F(\alpha, x)| \leq \epsilon \quad \text { for }(\alpha, x) \in S \times G .
$$

This definition is equivalent to the definition below.

Definition $1^{*}$. Equation (1) is said to be stable if there exists a function $\Phi:(0,+\infty) \rightarrow(0,+\infty)$ such that for every $\epsilon>0$ and every function $H: S \times$ $G \rightarrow S$ if

$$
|H(H(\alpha, x), y)-H(\alpha, x y)| \leq \Phi(\epsilon) \quad \text { for } \alpha \in S \text { and } x, y \in G,
$$

then there exists a solution $F$ of (1) such that

$$
|H(\alpha, x)-F(\alpha, x)| \leq \epsilon \quad \text { for } \alpha \in S \text { and } x \in G .
$$

This function $\Phi$ (the measure of stability), if it exists, is not unique since the function $a \Phi$ for every function $a:(0,+\infty) \rightarrow(0,1]$ is a measure of stability too. If there exists an unbounded measure of stability $\Phi$ (i.e., $\sup \Phi((0,+\infty))=+\infty)$, then the stability is said to be normal. This measure $\Phi$ is said to be maximal if it does not exist any measure of stability $\Phi_{1}$ for which $\Phi_{1}\left(\epsilon_{0}\right)>\Phi\left(\epsilon_{0}\right)$ for some $\epsilon_{0}>0$.

REMARK 1. Let $I(\epsilon)$ for $\epsilon>0$ be the set of $\delta>0$ such that for every function $H$ if $(2)$ is satisfied, then (3) is true for some solution $F$ of (1). If the equation (11) is stable, then $I(\epsilon) \neq \emptyset$ for every $\epsilon>0$. It is easy to see that $I(\epsilon)$ is an interval with $\inf I(\epsilon)=0$ (if $\delta_{0} \in I(\epsilon)$ and $0<\delta<\delta_{0}$, then $\delta \in I(\epsilon))$ and that $I\left(\epsilon_{1}\right) \subset I\left(\epsilon_{2}\right)$ for $\epsilon_{1}<\epsilon_{2}$. From here the equation (1) is normally stable if and only if the set $\bigcup_{\epsilon>0} I(\epsilon)$ is unbounded. If there exists an unbounded measure of stability, then there exists the bounded measure of stability. On the other hand if there exists a bounded measure of stability, then the unbounded measure of stability does not necessarily exist.

EXAMPLE 1. It is known that for the Cauchy's equation of the additive function $f(x+y)=f(x)+f(y)$, where $f: \mathbb{R} \rightarrow \mathbb{R}$, the function $\Phi(\epsilon)=\epsilon$ is a measure of stability. This measure is maximal. Indeed, assume that there exists a measure of stability $\Phi_{1}$ of this equation for which $\Phi_{1}(\epsilon)=\epsilon_{1}>\epsilon$ for any $\epsilon$. Then for every function $g: \mathbb{R} \rightarrow \mathbb{R}$ if $|g(x+y)-g(x)-g(y)| \leq \Phi_{1}(\epsilon)=\epsilon_{1}$, there exists an additive function $f$ such that $|g(x)-f(x)| \leq \epsilon$. Thus for 
$g(x)=\epsilon_{1}$ there exists an additive function $f$ such that $\left|\epsilon_{1}-f(x)\right| \leq \epsilon$. From here $f(x)=0$ as the bounded additive function, thus $\epsilon_{1} \leq \epsilon$ and we have a contradiction.

The maximal measure of stability needs not to exist, e.g., there is no maximal measure for the equation for which every function is the solution (see 2.12 in this paper).

Proposition 1. If equation (1) is stable, then there exists the maximal measure of stability of (1) if and only if $\sup I(\epsilon) \in I(\epsilon)$ for every $\epsilon>0$.

Proof. If $\sup I(\epsilon) \in I(\epsilon)$ for every $\epsilon>0$, then the function $\Phi(\epsilon)=$ $\sup I(\epsilon)$ is evidently the maximal measure of stability. If there exists an $\epsilon_{0}>0$ such that $\sup I\left(\epsilon_{0}\right)$ is not in $I\left(\epsilon_{0}\right)$ and $\Phi$ is a measure of stability, then $\Phi\left(\epsilon_{0}\right)<$ $\sup I\left(\epsilon_{0}\right)$. The function $\Phi_{1}$ such that $\Phi_{1}(\epsilon)=\Phi(\epsilon)$ for $\epsilon \neq \epsilon_{0}$ and $\Phi_{1}\left(\epsilon_{0}\right) \epsilon$ $\left(\Phi\left(\epsilon_{0}\right), \sup I\left(\epsilon_{0}\right)\right)$ is the measure of stability for which $\Phi\left(\epsilon_{0}\right)<\Phi_{1}\left(\epsilon_{0}\right)$.

Definition 2. Equation (1) is said to be $b$-stable if for every function $H: S \times G \rightarrow S$ such that the function $|H(H(\alpha, x), y)-H(\alpha, x y)|$ is bounded, there exists a solution $F$ of (1) such that the function $|H(\alpha, x)-F(\alpha, x)|$ is bounded too, i.e., if for every function $H: S \times G \rightarrow S$ such that there exists a $\delta>0$ for which (2) is satisfied, there exists an $\epsilon>0$ for which (3) is satisfied for a solution $F$ of (1).

If $a:=\inf \{|\alpha-\beta|: \alpha, \beta \in S, \alpha \neq \beta\}>0$, then equation (1) is stable, since it is enough to put $\delta<a$ in Definition 1. In this case equation (1) is not necessarily b-stable (see Example 1). Hence Definitions 1 and 2 are not equivalent.

The stability of the translation equation is considered in the papers [7, 11, 14, 18, 20, 21.

In the definition of b-stability $\epsilon$ depends on $\delta$ and on the function $H$. If $\epsilon$ does not depend precisely on the function $H$ the b-stability is called uniform. We have the following definition.

Definition 3. Equation (1) is said to be uniformly b-stable if for every $\delta>0$ there exists $\epsilon>0$ such that for every function $H: S \times G \rightarrow S$ which satisfies (2) there exists a solution $F$ of (1) such that (3) is satisfied.

This definition is equivalent to the definition below.

Definition $3^{*}$. Equation (1) is said to be uniformly b-stable if there exists a function $\Psi:(0,+\infty) \rightarrow(0,+\infty)$ such that for every $\delta>0$ and every function 
$H: S \times G \rightarrow S$ if

$$
|H(H(\alpha, x), y)-H(\alpha, x y)| \leq \delta \quad \text { for } \alpha \in S \text { and } x, y \in G,
$$

then there exists a solution $F$ of (1) such that

$$
|H(\alpha, x)-F(\alpha, x)| \leq \Psi(\delta) \quad \text { for } \alpha \in S \text { and } x \in G .
$$

The function $\Psi$ (the measure of uniform b-stability), if exists, is not unique since the function $a \Psi$ for every function $a:(0,+\infty) \rightarrow[1,+\infty)$ is "good" too. If there exists a measure of uniform b-stability $\Psi$ such that inf $\Psi((0,+\infty))=0$ (i.e., the function $1 / \Psi$ is unbounded), then the uniform b-stability is said to be normal. The measure $\Psi$ is said to be minimal if there is no measure of uniform b-stability $\Psi_{1}$ for which $\Psi_{1}\left(\delta_{0}\right)<\Psi\left(\delta_{0}\right)$ for any $\delta_{0}>0$.

REMark 2. Let $J(\delta)$ for $\delta>0$ be the set of $\epsilon>0$ such that for every function $H$ if $(2)$ is satisfied, then (3) is true for some solution $F$ of (1). If equation (1) is uniformly b-stable, then $J(\delta) \neq \emptyset$ for every $\delta>0$. It is easy to see that $J(\delta)$ is an interval with $\sup J(\delta)=+\infty$ and that $J\left(\delta_{1}\right) \subset J\left(\delta_{2}\right)$ for $\delta_{1}>\delta_{2}$. So equation (1) is normally uniformly b-stable if and only if $\inf \bigcup_{\delta>0} J(\delta)=0$.

If there exists a measure of uniform b-stability $\Psi$ such that $1 / \Psi$ is unbounded, then there exists a measure of uniform b-stability $\Psi_{1}$ such that $1 / \Psi_{1}$ is bounded. On the other hand if there exists a measure of uniform bstability $\Psi_{1}$ such that $1 / \Psi_{1}$ is bounded, then a measure of uniform b-stability $\Psi$ such that $1 / \Psi$ is unbounded does not necessarily exist.

The following proposition holds true and its proof is analogous to that of Proposition 1.

Proposition 2. If equation (1) is uniformly b-stable, then there exists the minimal measure of uniform b-stability of this equation if and only if $\inf J(\delta) \in J(\delta)$ for every $\delta>0$.

REMARK 3. The stability is in fact uniform since $\delta$ does not depend on the function $H$ in Definition 1 . The non uniform stability, i.e., the following property

for every $\epsilon>0$ and for every function $H: S \times G \rightarrow S$ there exists a $\delta>0$ such that if $(2)$ is satisfied, then there exists a solution $F$ of (1), for which (3) is satisfied, has no interest since every translation equation is stable in this sense.

Indeed, if the function $H$ is the solution of (1), then $\delta$ may be arbitrary. If not, there exist $\alpha_{0}, x_{0}, y_{0}$ such that $\left|H\left(H\left(\alpha_{0}, x_{0}\right), y_{0}\right)-H\left(\alpha_{0}, x_{0} y_{0}\right)\right| \neq 0$ 
and inequality (2) is not true for $\alpha_{0}, x_{0}, y_{0}$ and $\delta$ such that $0<\delta<$ $\left|H\left(H\left(\alpha_{0}, x_{0}\right), y_{0}\right)-H\left(\alpha_{0}, x_{0} y_{0}\right)\right|$.

REMARK 4. If the measure of stability $\Phi$ is a bijection, then this stability is normal and the function $\Phi^{-1}$ is a bijection too, thus the equation is normally uniformly b-stable. We have this situation for equation (1) if $S$ is an arbitrary metric space and the groupoid $G$ has only one element or it is $x y=x$ for $x, y \in G$ (here $\Phi(\alpha)=\alpha$ ) [16]. We have the same result for the measure of b-stability.

Proposition 3. If the stability of (1) is normal, then this equation is uniformly b-stable.

Proof. Indeed, assume that the function $|H(H(\alpha, x), y)-H(\alpha, x y)|$ is bounded by $\delta$. There exists an $\epsilon$ such that $\Phi(\epsilon) \geq \delta$ and since (1) is normally stable, then there exists a solution $F$ of (1) such that $|H(\alpha, x)-F(\alpha, x)| \leq \epsilon$, thus $|H(\alpha, x)-F(\alpha, x)|$ is bounded.

ExAmple 2. Equation (1) with $S=\mathbb{Z}$ with the usual metric and with $G$ as the free group generated by two elements $a, b$ is not b-stable [15]. This equation is stable since $\inf \{|\alpha-\beta|: \alpha, \beta \in \mathbb{Z}, \alpha \neq \beta\}=1>0$. It is not normally stable by Proposition 1 .

It is possible to give the proof of this fact without using the result in [15]. Suppose that there exists the unbounded measure of stability $\Phi$. Thus there exists an $\epsilon>0$ such that $\Phi(\epsilon) \geq 1$. Assume that the element $x \in G$ is reduced, i.e., in $x$ do not appear the pairs of the form $a a^{-1}, a^{-1} a, b b^{-1}, b^{-1} b$ and it is noted by the power of $a$ and $b$ only with the indices 1 or -1 . Let $g: G \rightarrow \mathbb{Z}$ be the function such that $g(x)=r(x)-s(x)$, where $r(x)$ is the number of pairs $a b$ in $x$ and $s(x)$ is the number of pairs $b^{-1} a^{-1}$ in $x$. We have $g(x y)-g(x)-g(y) \in\{-1,0,1\}$ (see [8] and [1]). If $H(\alpha, x)=\alpha+g(x)$, then $H(H(\alpha, x), y)-H(\alpha, x y) \in\{-1,0,1\}$, thus $|H(H(\alpha, x), y)-H(\alpha, x y)| \leq 1 \leq$ $\Phi(\epsilon)$. Then there exists a solution $F$ of (1) such that $|H(\alpha, x)-F(\alpha, x)| \leq \epsilon$. We have for $n \in \mathbb{Z}$

$$
F\left(\alpha, a^{n}\right)-H\left(\alpha, a^{n}\right)=F\left(\alpha, a^{n}\right)-\alpha-g\left(a^{n}\right)=F\left(\alpha, a^{n}\right)-\alpha .
$$

Thus the set $S^{*}=\left\{F\left(\alpha, a^{n}\right)-\alpha: \alpha, n \in \mathbb{Z}\right\}$ is bounded. The function $K(\alpha, n)=F\left(\alpha, a^{n}\right)$ is a solution of the equation

$$
K(K(\alpha, n), m)=K(\alpha, n+m) .
$$


Thus it is of the form (see [14])

$$
K(\alpha, n)=h_{k}^{-1}\left(h_{k}(f(\alpha))+n\right) \quad \text { for } f(\alpha) \in S_{k},
$$

where $f: \mathbb{Z} \rightarrow \mathbb{Z}$ is the function such that $f(f)=f, f(\mathbb{Z})=\bigcup_{k \in K} S_{k}, S_{k} \neq \emptyset$, $S_{k} \cap S_{l}=\emptyset$ for $k \neq l$ and for every $k \in K$ there exists the subgroup $\mathbb{Z}_{k}$ of the group $\mathbb{Z}$ such that card $S_{k}=$ ind $\mathbb{Z}_{k}$ and $h_{k}: S_{k} \rightarrow \mathbb{Z} / \mathbb{Z}_{k}$ is a bijection.

Let $\alpha \in \mathbb{Z}$ be fixed and $f(\alpha) \in S_{k}$. If $\mathbb{Z}_{k}=\{0\}$, then the function $K(\alpha, \cdot): \mathbb{Z} \rightarrow S_{k}$ is an injection, thus the set $\{K(\alpha, n): n \in \mathbb{Z}\}$ is unbounded as infinite $(S=\mathbb{Z})$. We have a contradiction since this set is a subset of $S^{*}$. There exists the element $p>0$ in $S_{k}$, thus $F\left(\alpha, a^{p}\right)=K(\alpha, p)=f(\alpha)$. By the analogous consideration for $b$ in place of $a$ we obtain that there exists the element $q>0$ such that $F\left(\alpha, b^{q}\right)=f(\alpha)$. We put $A=\left\{\left(a^{p} b^{q}\right)^{n}: n \in \mathbb{Z}, n>0\right\}$. Since $F$ is the solution of (1) we have $F(\alpha, x)=f(\alpha)$ for $x \in A$. Moreover, $g\left(\left(a^{p} b^{q}\right)^{n}\right)=n$, thus the function

$$
F\left(\alpha,\left(a^{p} b^{q}\right)^{n}\right)-\alpha-g\left(\left(a^{p} b^{q}\right)^{n}\right)=f(\alpha)-\alpha-n,
$$

as the function of $n$, is unbounded. We have a contradiction since the function $|H(\alpha, x)-F(\alpha, x)|$ is bounded.

Proposition 4. If the b-stability of (1) is uniform and normal, then this equation is normally stable.

Proof. There exists a measure of b-stability $\Psi$ which satisfies the condition $\inf \Psi((0,+\infty))=0$. From here for every $\epsilon>0$ there exists a $\delta>0$ such that $\Psi(\delta) \leq \epsilon$. Assume that we have $|H(H(\alpha, x), y)-H(\alpha, x y)| \leq \delta$. Then we obtain $|H(\alpha, x)-F(\alpha, x)| \leq \Psi(\delta) \leq \epsilon$ for some solution $F$ of (1).

We consider the stability of (1) in an arbitrary class $C$ of functions from $S \times G$ to $S$ when the functions $H$ and $F$ are only in the class $C$.

B. Przebieracz has proved in [22], by using the result of J. Chudziak [7], the following Ulam-Hyers stability theorem of equation (1) in the class of continuous functions.

TheOREM. Let $I \subset \mathbb{R}$ be an interval. Suppose that $H: I \times \mathbb{R} \rightarrow I$ is continuous with respect to each variable and satisfies

$$
|H(H(\alpha, x), y)-H(\alpha, x+y)| \leq \delta \quad \text { for } \alpha \in I \text { and } x, y \in \mathbb{R}
$$

Then there exists a continuous solution $F$ of the equation

$$
F(F(\alpha, x), y)=F(\alpha, x+y)
$$


such that

$$
|H(\alpha, x)-F(\alpha, x)| \leq 10 \delta \quad \text { for } \alpha \in I \text { and } x \in \mathbb{R}
$$

This result, in this formulation, is in fact the statement that equation (5) is normally uniformly b-stable in the class $C$ of continuous functions, here $\Psi(\delta)=10 \delta$. However, from here we get that equation (5) is also normally stable in the class $C$ and $\Phi(\epsilon)=\epsilon / 10$. This class $C$ is natural for the applications of translation equation in dynamical systems or iteration groups.

ExAmple 3. Equation (1) with $S=[0,1]$ with the usual metric and with $G$ as the group of two elements $e, a$ ( $e$ is the unity element) is not stable in the class $C$ of the functions $H: S \times G \rightarrow S$ such that $H(\alpha, e)=\alpha$ for $\alpha \in S$ [16]. This class $C$ is natural for those applications of translation equation, in which there exists the unit $e$ in the structure $G$. The equation in consideration is uniformly b-stable (the function $\Psi(\delta)=1$ is "good" for Definition $3^{*}$ ). This b-stability is not normal by Proposition 2 .

It is possible to transfer the above considerations on systems of the functional equations. We show this transfer for the dynamical system. The continuous function $F: I \times \mathbb{R} \rightarrow I$, where $I \subset \mathbb{R}$ is an interval with nonempty interior, is said to be a dynamical system if $F$ satisfies equation (5) and

$$
F(\alpha, 0)=\alpha \text { for every } \alpha \in I \text {. }
$$

This condition is for the solutions $F$ of (5) equivalent to the condition

$$
F^{\prime}(\alpha, 0)=1 \quad \text { for } \alpha \in I \text {. }
$$

In [19] the following Corollary 3.8 is proved:

COROLlary. If the continuous function $H: I \times \mathbb{R} \rightarrow I$ satisfies inequality (4) for some $\delta \in\left(0, \frac{2}{5}\right)$ and $\left|H^{\prime}(\alpha, 0)-1\right| \leq \delta$ for $\alpha \in I$, then there exists a dynamical system $F$ such that (6) is true.

For $\epsilon>0$ let $\Phi(\epsilon)=\frac{1}{2} \min \left\{\frac{\epsilon}{5}, \frac{2}{5}\right\}$. If (4) with $\delta=\Phi(\epsilon)$ is satisfied and $\left|H^{\prime}(\alpha, 0)-1\right| \leq \Phi(\epsilon)$ is true for $\alpha \in I$, then by the above corollary $\mid H(\alpha, x)-$ $F(\alpha, x) \mid \leq 10 \Phi(\epsilon)=\min \{\epsilon, 2\} \leq \epsilon$ for some dynamical system $F$. Hence the function $\Phi$ is a measure of stability of the system and the function $\Phi$ is bounded.

The stability in consideration is not normal for $I$ unbounded since in this case the system (5) and (7) is not b-stable. Indeed, for $H(\alpha, x)=\alpha_{0}$ for an $\alpha_{0} \in I$ we have $|H(H(\alpha, x), y)-H(\alpha, x+y)|=0$ for $\alpha \in I, x, y \in \mathbb{R}$ and 
$\left|H^{\prime}(\alpha, 0)-1\right|=1$ for $\alpha \in I$. Moreover, for every dynamical system $F$ the function $|H(\alpha, 0)-F(\alpha, 0)|=\left|\alpha_{0}-\alpha\right|$ is unbounded.

For the interval $I$ bounded the system in consideration is normally uniformly b-stable since the function $\Psi(\delta)=10 \delta$ for $0<\delta<\frac{2}{5}$ and $\Psi(\delta)=|I|$ (the length of $I$ ) for $\delta \geq \frac{2}{5}$ is the measure of uniform b-stability of our system and the function $1 / \Psi$ is unbounded. Our system is normally stable too by the function $\Phi(\epsilon)=\frac{1}{2} \min \left\{\frac{\epsilon}{5}, \frac{2}{5}\right\}$ for $0<\epsilon<|I|$ and $\Phi(\epsilon)=\epsilon$ for $\epsilon \geq|I|$.

Correction to the paper [19]. By the above the phrase "... as well as uniformly b-stability (hence b-stability)" as the conclusion of [19, Corollary 3.8] after the proof of this corollary in the paper [19, p. 290] is not true for $I$ unbounded (my mistake). Hence in the table in the end of the paper [19] (see [23] too) for the b-stability and uniform b-stability in the case of def. 2 in place of "for every I" must be "only for I bounded". The table must be thus of the form

\begin{tabular}{|c|c|c|c|c|c|}
\hline & $\begin{array}{c}\text { def. } 1 \\
(0,1) \& \\
F(0, x)=x\end{array}$ & $\begin{array}{c}\text { def. } 2 \\
(0,1) \& \\
F(0, x)=1\end{array}$ & $\begin{array}{c}\text { def. } 3 \\
(0,1) \& F(0, \cdot) \\
\text { strictly } \\
\text { increasing }\end{array}$ & $\begin{array}{c}\text { def. } 4 \\
(0,1) \& \\
F^{\prime}(0, x) \\
\text { exists }\end{array}$ & $\begin{array}{c}\text { def. } 5 \\
(0,1) \& \\
F \text { surjection }\end{array}$ \\
\hline $\begin{array}{l}\text { Ulam-Hyers } \\
\text { stability }\end{array}$ & only for $I=\mathbb{R}$ & for every $I$ & for no & & for every $I$ \\
\hline b-stability & only for $I$ & \multirow{2}{*}{\multicolumn{3}{|c|}{ only for $I$ bounded }} & \\
\hline $\begin{array}{l}\text { uniform } \\
\text { b-stability }\end{array}$ & $\begin{array}{l}\text { bounded or } \\
I=\mathbb{R}\end{array}$ & & & & for every $I$ \\
\hline $\begin{array}{l}\text { inverse } \\
\text { stability }\end{array}$ & \multicolumn{5}{|c|}{ for no $I$} \\
\hline $\begin{array}{l}\text { inverse } \\
\text { b-stability }\end{array}$ & \multirow{2}{*}{$\begin{array}{l}\text { only for } I \\
\text { bounded }\end{array}$} & \multirow[b]{2}{*}{ for no $I$} & \multirow{2}{*}{\multicolumn{3}{|c|}{ only for $I$ bounded }} \\
\hline $\begin{array}{l}\text { inverse uniform } \\
\text { b-stability }\end{array}$ & & & & & \\
\hline superstability & \multicolumn{5}{|c|}{ only for $I$ bounded } \\
\hline $\begin{array}{l}\text { inverse } \\
\text { superstability }\end{array}$ & $\begin{array}{l}\text { only for } I \\
\text { bounded }\end{array}$ & for no $I$ & \multicolumn{3}{|c|}{ for every $I$} \\
\hline hyperstability & \multicolumn{5}{|c|}{ for no $I$} \\
\hline $\begin{array}{l}\text { inverse } \\
\text { hyperstability }\end{array}$ & \multicolumn{5}{|c|}{ for every $I$} \\
\hline
\end{tabular}

Here $(0,1)$ signifies the equation (5) in the form $F(s, F(t, x))=F(t+s, x)$, where $F: \mathbb{R} \times I \rightarrow I$.

In this table the results on the different stabilities for the five equivalent definitions of the dynamical system in the class of continuous functions are presented.

Conclusion 1. Let $C_{1}$ be the class of the functions from $I \times \mathbb{R}$ to $I$ and $C_{2}$ the class of continuous functions in $C_{1}$. The system of functional equations (5) and (7) is normally stable in the class $C_{2}$. This system is normally uniformly b-stable in the class $C_{1}$ (in the class $C_{2}$ ) if and only if the interval I is bounded. 
Remark 5. We can consider the restricted uniform b-stability if $\delta$ in Definitions 3 and $3^{*}$ is limited to $\delta<\delta_{0}$ for some $\delta_{0}>0$ (there exists a $\delta_{0}>0$ such that for every $\left.\delta<\delta_{0} \ldots\right)$. Here the measure of restricted uniform b-stability is the function $\Psi$ from $\left(0, \delta_{0}\right)$ to $(0,+\infty)$ and this b-stability is normal if the function $1 / \Psi$ is unbounded. By the above considerations the system of equations (5) and (7) is restrictly uniformly b-stable for $\delta_{0}=\frac{2}{5}$ even for the unbounded interval $I$ and this b-stability is normal $(\Psi(\delta)=10 \delta)$. The translation equation in Example 2 is normally restrictly uniformly b-stable for $\delta_{0}=1$ (e.g., $\Psi(\delta)=\delta)$.

The number $\frac{2}{5}$ can be replaced in [19, Corollary 3.8] and in the above by 1 . In this case the estimation of the function $|H(\alpha, x)-F(\alpha, x)|$ in [19, Corollary $3.8]$ is $\delta \max \left\{6+\frac{2+\delta}{1-\delta}, 9\right\}$ and it tends to $+\infty$ if $\delta \rightarrow 1-0$.

We notice that the restricted stability (there exists $\epsilon_{0}>0$ such that for every $\left.\epsilon<\epsilon_{0} \ldots\right)$ is equivalent to stability.

We complete the above table by the three lines for: restricted b-stability, normal stability and normal uniform b-stability.

\begin{tabular}{|c|c|c|c|c|}
\hline & def. 1 & def. 2 & \begin{tabular}{l|l} 
def. 3 & def. 4
\end{tabular} & def. 5 \\
\hline $\begin{array}{l}\text { restricted uniform } \\
\text { b-stability (normal) }\end{array}$ & $\begin{array}{c}\text { only for } I \\
\text { bounded or } I=\mathbb{R}\end{array}$ & for every $I$ & $\begin{array}{l}\text { only for } I \\
\text { bounded }\end{array}$ & for every $I$ \\
\hline normal stability & only for $I=\mathbb{R}$ & $\begin{array}{c}\text { only for } I \\
\text { bounded }\end{array}$ & for no $I$ & for every $I$ \\
\hline $\begin{array}{l}\text { normal uniform } \\
\text { b-stability }\end{array}$ & $\begin{array}{c}\text { only for } I \\
\text { bounded or } I=\mathbb{R}\end{array}$ & $\begin{array}{c}\text { only for } I \\
\text { bounded }\end{array}$ & $\begin{array}{c}\text { only for } I \\
\text { bounded }\end{array}$ & for every $I$ \\
\hline
\end{tabular}

\section{PROOF FOR RESTRICTED UNIFORM B-STABILITY.}

1) The uniformly b-stable dynamical system is evidently restrictly uniformly b-stable (but not inversely).

2) If in the proof of Theorem 4.3 in [19] we assume that $2 b-2 a \leq \delta$ for a $\delta>0$, then we obtain the counter-example for the restricted uniform b-stability in the case of the def. 1 and if the unbounded interval $I$ is bounded from below (analogous from above).

3) For def. 2. The restricted uniform b-stability for every $I$ in this case is proved above.

4) For def. 3 and def. 4 . The function $H(t, x)=\frac{\delta}{\pi} \arctan [x-(a+b)]+(a+b)$ for $x, 2 a, 2 b \in I, a<b, 0<\delta \leq 2 b-2 a$, is strictly increasing, differentiable function from $I$ to the interval $\left[(a+b)-\frac{\delta}{2},(a+b)+\frac{\delta}{2}\right] \subset[2 a, 2 b] \subset I$ (def.3 and def. 4 in the table). Thus $|H(s, H(t, x))-H(t+s, x)| \leq \delta$. If the interval $I$ is unbounded, then there does no exist the dynamical system $F$ for which $|H(0, x)-F(0, x)|=|H(0, x)-x|$ is bounded.

The above restricted uniform b-stability is normal. For definitions 1, 3, 4, 5 this is the conclusion from the fact that the normal uniform b-stability implies 
evidently the normal restricted uniform b-stability. For def. 2 the function $\Psi(\delta)=10 \delta:\left(0, \frac{2}{5}\right) \rightarrow(0,+\infty)$ is the measure for which $\inf \Psi\left(\left(0, \frac{2}{5}\right)\right)=0$ (see [19, Corollary 3.8]).

PROOF FOR NORMAL STABILITY.

1) For def. 1. Here the measure of stability is $\Phi(\epsilon)=\frac{\epsilon}{9}[22]$.

2) For def. 2. We have in this case the stability for every $I$ thus there exists the measure of stability $\Phi_{1}$ (not necesssarily unbounded). The function $\Phi(x)=\Phi_{1}(x)$ for $0<x \leq|I|$ (the length of $I$ ) and $\Phi(x)=x$ for $x>|I|$ is the unbounded measure by the definition of the measure. This stability is not normal for $I$ unbounded. Indeed, assume that there exists the measure of stability $\Phi$ of the dynamical system by def. 2 such that $\Phi(\epsilon) \geq 1$ for any $\epsilon>0$. The function $H(t, x)=c$ for every $(t, x) \in \mathbb{R} \times I$ and a $c \in I$ satisfies the inequalities

$$
|H(s, H(t, x))-H(s+t, x)|=0 \leq \Phi(\epsilon) \quad \text { and } \quad\left|H^{\prime}(0, x)-1\right|=1 \leq \Phi(\epsilon),
$$

thus there exists a dynamical system $F$ such that $|H(t, x)-F(t, x)| \leq \epsilon$. Since $F(0, x)=x$, hence $|c-x| \leq \epsilon$ and we have a contradiction.

$3)$ For def. 3 and def. 4 . The dynamical system is not stable thus it is not normally stable too.

4) For def. 5 see 1$)$.

ProOF FOR NORMAL UNIFORM B-STABILITY.

1) For def. 1. For $I=\mathbb{R}$ the function $\Psi(\delta)=9 \delta$ is "good" [22]. For I bounded the function $\Psi(\delta)=10 \delta$ for $\delta \in\left(0, \frac{2}{5}\right)$ and $\Psi(\delta)=|I|$ (the length of $I$ ) for $\delta \in\left[\frac{2}{5},+\infty\right)$ is the measure of b-stability for which $\inf \Psi(0,+\infty)=0$. Indeed, if $\delta \in\left(0, \frac{2}{5}\right)$, then by [19, Corollary 3.8] for every function $H: \mathbb{R} \times$ $I \rightarrow I$ such that

$$
|H(s, H(t, x))-H(s+t, x)| \leq \delta \quad \text { and } \quad\left|H^{\prime}(0, x)-1\right| \leq \delta
$$

for $(t, x) \in \mathbb{R} \times I$, there exists a solution $F$ of our dynamical system such that $\mid H(t, x)-F(t, x)) \mid \leq \Psi(\delta)$ for $(t, x) \in \mathbb{R} \times I$. If $\delta \in\left[\frac{2}{5},+\infty\right)$, then $|H(t, x)-F(t, x)| \leq|I|=\Psi(\delta)$ for $(t, x) \in \mathbb{R} \times I$ and all functions $H, F: I \times$ $\mathbb{R} \rightarrow I$.

This normal uniform b-stability is only for $I=\mathbb{R}$ or $I$ bounded since the b-stability in this case is only for $I=\mathbb{R}$ or $I$ bounded.

2) For def. 2, def. 3 and def. 4 . See above. This normal uniform b-stability is only for $I$ bounded since the b-stability in this case is only for $I$ bounded.

3) For def. 5. In this case the function $\Psi(\delta)=9 \delta$ is "good" [22]. 


\section{Other functional equations}

The definitions and the propositions above may be formulated by the natural way for arbitrary functional equations. We give some examples of this reformulation.

2.1. If in the differential equation

$$
f^{\prime}(x)=H(f(x))
$$

(the autonomous differential equation), where $f:[a, b] \rightarrow \mathbb{R}$, the function $H: \mathbb{R} \rightarrow \mathbb{R}$ is such that $|H(u)-H(v)| \leq L|u-v|$ for $u, v \in \mathbb{R}$ and $L(b-a)<1$ for some $L$, then this equation is normally stable and normally uniformly b-stable.

Indeed, let $g: \mathbb{R} \rightarrow \mathbb{R}$ be such that

$$
\left|g^{\prime}(x)-H(g(x))\right| \leq \delta
$$

for a $\delta>0$ and $x \in[a, b]$. Thus we have

$$
\left|g(x)-g(a)-\int_{a}^{x} H(g(t)) d t\right| \leq \delta(b-a) .
$$

Let $f$ be the solution of (8) such that $f(a)=g(a)$, thus

$$
f(x)-f(a)-\int_{a}^{x} H(f(t)) d t=0,
$$

therefore

$$
\left|g(x)-f(x)-\int_{a}^{x}[H(g(t))-H(f(t))] d t\right| \leq \delta(b-a) .
$$

We obtain

$$
\begin{aligned}
|g(x)-f(x)| & \leq\left|\int_{a}^{x}[H(g(t))-H(f(t))] d t\right|+\delta(b-a) \\
& \leq\left|\int_{a}^{x} L[g(t)-f(t)] d t\right|+\delta(b-a) \\
& \leq(b-a) L \sup _{x \in[a, b]}|g(x)-f(x)|+\delta(b-a) .
\end{aligned}
$$


Hence

$$
|g(x)-f(x)| \leq[1-L(b-a)]^{-1}(b-a) \delta .
$$

For the unbounded interval $I$ equation $(8)$ is neither stable nor b-stable. E.g., for the equation $f^{\prime}(x)=1$ for $f: I \rightarrow \mathbb{R}$ the function $g(x)=(1+\delta) x$ for $\delta>0$ is such that $\left|g^{\prime}(x)-1\right| \leq \delta$ and $|g(x)-(x+c)|=|\delta x-c|$ is unbounded.

It is well-known (e.g., see [24]) the relation between equation (8) and the translation equation. If the function $H: \mathbb{R} \rightarrow \mathbb{R}$ is different from 0 and satisfies the Lipschitz's condition (the difference quotient of $H$ is bounded), then the solution of equation (8) for which $f(0)=\alpha$ is of the form $f(x)=G^{-1}[x+$ $G(\alpha)]$, where $G(y)=\int \frac{1}{H(y)} d y$. The function $F(\alpha, x)=G^{-1}[x+G(\alpha)]$ is in this case the solution of the translation equation (5) for which $F(\alpha, 0)=\alpha$, thus it is the dynamical system. From the above table this dynamical system (i.e., the system of the equations which determines this dynamical system) is stable only for $I=\mathbb{R}$ (from [22] the measure $\Phi(\epsilon)=\frac{\epsilon}{9}$, thus this stability is normal) and it is uniformly b-stable only for $I$ bounded (here this b-stability is not normal by the proposition for dynamical systems analogous to Proposition 4 ) or $I=\mathbb{R}$ (from [22] the measure $\Psi(\delta)=9 \delta$, thus this b-stability is normal too). And vice versa. If $F(\alpha, x)=G^{-1}[x+G(\alpha)]$ and $G^{\prime}(y) \neq 0$, then $f(x)=$ $G^{-1}[x+G(\alpha)]$ for $\alpha$ fixed is the solution of the equation $f^{\prime}(x)=\left[\frac{1}{G^{\prime}(y)}\right]_{y=f(x)}$ such that $f(0)=\alpha$.

For the above function $F(\alpha, x)=G^{-1}[x+G(\alpha)]$ there exists an $\alpha_{0}$ such that $F\left(\alpha_{0}, x\right)$ is a continuous surjection of $\mathbb{R}$ onto $I$ (in fact every $\alpha_{0}$ in $I$ is "good" here). By [7] equation (5) is for every interval $I$ normally stable with $\Phi(\epsilon)=\frac{\epsilon}{9}$ and normally uniformly b-stable with $\Psi(\delta)=9 \delta$ in the class $C$ of the functions $H: I \times \mathbb{R} \rightarrow I$ such that for every function $H \in C$ there exists $\alpha_{0} \in I$ for which $H\left(\alpha_{0}, x\right)$ is the continuous surjection of $\mathbb{R}$ to $I$.

2.2. The equation

$$
f(\alpha, x)+f(f(\alpha, x)+\alpha, y)=f(\alpha, x+y)
$$

for $f: \mathbb{R} \times \mathbb{R} \rightarrow \mathbb{R}$ has applications [1] in the theory of Jabotinsky's equations [12. For every $\delta \geq 0$ the function $g: \mathbb{R} \times \mathbb{R} \rightarrow \mathbb{R}$ is the solution of the inequality

$$
|g(\alpha, x)+g(g(\alpha, x)+\alpha, y)-g(\alpha, x+y)| \leq \delta
$$

if and only if the function $G(\alpha, x)=g(\alpha, x)+\alpha$ is the solution of the inequality

$$
|G(G(\alpha, x), y)-G(\alpha, x+y)| \leq \delta
$$


From here the equation in consideration is in the class of continuous functions normally stable (with $\delta=\epsilon / 10$ ) and normally uniformly b-stable by the result of B. Przebieracz in [22].

2.3. The exponential Cauchy equation

J.A. Baker, J. Lawrence and F. Zorzitto have proved in [2] that for the solution $g: \mathbb{R} \rightarrow(0,+\infty)$ of the inequality $|g(x+y)-g(x) g(y)| \leq \delta$ we have either $|g(x)| \leq \max \{4,4 \delta\}$ or $g$ is the solution of the equation $f(x+y)=$ $f(x) f(y)$. Thus this equation is uniformly b-stable. Let us assume that this equation is stable. Then for $\epsilon=1 / 2$ there exists a $\delta_{0}$ such that for any function $g: \mathbb{R} \rightarrow(0,+\infty)$ if $|g(x+y)-g(x) g(y)| \leq \delta_{0}$, then there exists a solution $f$ of the equation such that $|g(x)-f(x)| \leq 1 / 2$. This implication is satisfied for all positive $\delta \leq \delta_{0}$, too. For $g(x)=(1-\sqrt{1-4 \delta}) / 2$, where $0<\delta \leq \min \left\{1 / 4, \delta_{0}\right\}$, we have $g(x+y)-g(x) g(y)=\delta$, thus there exists a solution $f$ of the equation in consideration such that $|g(x)-f(x)| \leq 1 / 2$. The function $g$ is bounded, hence also $f$ must be bounded too, thus $f(x)=1$. Since

$$
1 / 2 \geq|(1-\sqrt{1-4 \delta}) / 2-1| \rightarrow 1 \quad \text { for } \delta \rightarrow 0
$$

we obtain a contradiction. Thus the equation in consideration is not stable and, as a consequence of this, it is not normally uniformly b-stable.

2.4. The Dhombres equation

B. Batko proved in [4] for the Dhombres equation

$$
[f(x)+f(y)][f(x+y)-f(x)-f(y)]=0,
$$

where $f$ is a function from abelian group $G$ to $\mathbb{C}$, that if $\mid[g(x)+g(y)][g(x+y)-$ $g(x)-g(y)] \mid \leq \delta$ for some $\delta>0$ and a function $g: G \rightarrow \mathbb{C}$, then $|g(x)| \leq \sqrt{\delta / 2}$ or $g$ is additive. Hence it is a solution of (10). Thus the equation (10) is normally uniformly b-stable $(\Psi(\delta)=\sqrt{\delta / 2})$ and it is normally stable $(\Phi(\epsilon)=$ $\left.2 \epsilon^{2}\right)$.

2.5. The cosine d'Alembert equation

J.A. Baker proved in [3] for the cosine d'Alembert equation

$$
f(x+y)+f(x-y)=2 f(x) f(y),
$$

where $f$ is a function from abelian group $G$ to $\mathbb{C}$, that if $\mid g(x+y)+g(x-$ $y)-2 g(x) g(y) \mid \leq \delta$ for a $\delta>0$ and a function $g: G \rightarrow C$, then $|g(x)| \leq$ $(1+\sqrt{1+2 \delta}) / 2$ or the function $g$ is a solution of the equation (11). Thus this equation is uniformly b-stable $(\Psi(\delta)=(1+\sqrt{1+2 \delta}) / 2)$. Infimum of the range of the above function $\Psi$ is not 0 and from here the question: is equation (11) normally uniformly b-stable? 
2.6. In [18] it is proved that the equation

$$
f^{2}(2 x)=4 f^{2}(x)
$$

where $f: \mathbb{R} \rightarrow \mathbb{R}$, is normally stable by the measure of stability $\Phi(\epsilon)=3 \epsilon^{2}$, thus it is normally uniformly b-stable with the function $\Psi(\delta)=\sqrt{\delta / 3}$.

2.7. The squares of Cauchy and Jensen equations

In [18] are proved the following theorems.

TheOrem. Let $G$ be a semigroup divisible by 2 and let $A$ be an algebra with multiplicative norm. Then for every bounded solution $g: G \rightarrow A$ of the inequality

$$
\left|[g(x+y)]^{2}[g(x)+g(y)]^{2}\right| \leq \delta,
$$

where $\delta \geq 0$, we have

$$
|g(x)| \leq \sqrt{\delta / 3} \quad \text { for } x \in G
$$

Since every unbounded solution of the inequality (12) is additive [18, the equation

$$
[f(x+y)]^{2}=[f(x)+f(y)]^{2}
$$

is normally stable and normally uniformly b-stable.

TheOrem. Let $G$ be an abelian semigroup and $A=\mathbb{R}$ or $A=\mathbb{C}$. If the function $[g(x+y)-g(x)]^{2}-g^{2}(y)$, for $g: G \rightarrow A$, is bounded, then $g$ is bounded or it is the solution of the equation

$$
[f(x+y)-f(x)]^{2}=f^{2}(y)
$$

From here equation (13) is b-stable. Is this equation uniformly b-stable (normally uniformly b-stable)?

We have the same situation for the equation

$$
[f(x+y)]^{2}=[f(x) f(y)]^{2},
$$

where $f: G \rightarrow A$, for $G$ and $A$ as above, and if the group $G$ is divisible by 2 for the equation of Jensen

$$
\left[f\left(\frac{x+y}{2}\right)\right]^{2}=\left[\frac{f(x)+f(y)}{2}\right]^{2}
$$


2.8. The Lobachevski's equation

P. Gavruta proved in [9] that if

$$
\left|g^{2}\left(\frac{x+y}{2}\right)-g(x) g(y)\right| \leq \delta
$$

for $x, y \in G$ and $g: G \rightarrow \mathbb{C}$, where $G$ is an abelian group uniquely divisible by 2 , then

$$
|g(x)| \leq \frac{|g(0)|+\sqrt{|g(0)|^{2}+4 \delta}}{2} \text { for } x \in G
$$

or $g$ is a solution of Lobachevski's equation, i.e.,

$$
g^{2}\left(\frac{x+y}{2}\right)=g(x) g(y)
$$

Thus this equation is b-stable and since $\frac{|g(0)|+\sqrt{|g(0)|^{2}+4 \delta}}{2}$ depends on the function $g$ (by $g(0)$ ) thus we have the question: is the Lobachevski's equation uniformly b-stable (normally uniformly b-stable)?

The function $g_{a}(x)$ for $a \geq 0$ such that $g_{a}(x)=\sqrt{a^{2}+\delta}$ for $x \in \mathbb{R} \backslash\{0\}$ and $g_{a}(0)=a$ is the solution of the inequality (14) and the family $\left\{g_{a}(x)\right\}_{a \geq 0}$ is not commonly bounded. This fact does not prove that equation 115 is not uniformly b-bounded. Indeed, for every function $g_{a}$ there exists the solution $f_{a}$ of 15 , namely $f_{a}(x)=\frac{a+\sqrt{a^{2}+\delta}}{2}$, such that

$$
\left|g_{a}(x)-f_{a}(x)\right|=\sqrt{a^{2}+\delta}-a \leq \sqrt{\delta} \text { for every } a \geq 0 .
$$

2.9. The sine equation

We have the same situation for the sine equation

$$
f(x+y) f(x-y)=f^{2}(x)-f^{2}(y) .
$$

P.W. Cholewa proved in [6] that for every function $g$ from the abelian group $G$ uniquely divisible by 2 to $\mathbb{C}$ if

$$
\left|g(x+y) g(x-y)-g^{2}(x)+g^{2}(y)\right| \leq \delta \quad \text { for } x, y \in G \text { and some } \delta>0,
$$

then $g$ is bounded or it is the solution of $(16)$. Thus equation 16 is b-stable. Is this equation uniformly b-stable?

P.W. Cholewa has remarked that the functions $g_{n}(x)=n \sin x+\frac{1}{n}$ for $n \in$ $\mathbb{N}$ satisfy inequality (17) for $\delta=3$ and the family $g_{n}(x)_{n \in \mathbb{N}}$ is not commonly bounded. This fact is not the proof that equation (16) is not uniformly b-stable 
since for every function $g_{n}$ there exists the solution $f_{n}$, namely $f_{n}(x)=n \sin x$, such that $\left|g_{n}(x)-f_{n}(x)\right|=\frac{1}{n} \leq 1$ for every $n \in \mathbb{N}$.

2.10. The Pexider's equation

We have as a corollary of a K. Nikodem's result [20] the following theorem.

TheOrem. Let $G$ be an abelian semigroup with zero and $B$ a Banach space. If the functions $f, g, h: G \rightarrow B$ are such that $|f(x+y)-g(x)-h(y)| \leq \delta$ for every $x, y \in G$ and some $\delta>0$, then there exist the functions $f_{1}, g_{1}, h_{1}: G \rightarrow$ $B$ such that $f_{1}(x+y)=g_{1}(x)+h_{1}(y)$ (the Pexider's equation) and $\mid f(x)-$ $f_{1}(x)|\leq 3 \delta| g,(x)-g_{1}(x)|\leq 4 \delta| h,(x)-h_{1}(x) \mid \leq 4 \delta$ for $x \in G$.

So the Pexider's equation is normally uniformly b-stable with $\Psi(\delta)=4 \delta$, thus normally stable too.

2.11. The quadratic equation

The quadratic equation

$$
f(x+y)+f(x-y)=2 f(x)+2 f(y),
$$

where $f$ is the function from an abelian group to a Banach space, is normally uniformly b-stable with $\Psi(\delta)=\delta / 2([6])$, thus it is normally stable too.

2.12. The system of the functions

$$
f(f(x))=f(x) \text { and } \quad f^{\prime}(x)=1,
$$

for $f: \mathbb{R} \rightarrow \mathbb{R}$, is not b-stable and it is restrictly b-stable and stable. Indeed, for the function $g(x)=0$ we have $|g(g(x))-g(x)| \leq 1$ and $\left|g^{\prime}(x)-1\right| \leq 1$ and $|g(x)-x|$ is not bounded. Assume that for a function $g: \mathbb{R} \rightarrow \mathbb{R}$ we have $|g(g(x))-g(x)| \leq \delta$ and $\left|g^{\prime}(x)-1\right| \leq \delta$ for a positive $\delta<1$. For here $0<1-\delta \leq$ $g^{\prime}(x) \leq 1+\delta$, the function $g$ is thus strictly increasing. We prove that this function is unbounded from above and from below. Suppose, for the indirect proof, that this function is bounded from above, thus the sequence $g(n)$, as increasing and bounded, is convergent. Since $g(n+1)-g(n) \rightarrow 0$ thus there exists a number $n$ such that $g(n+1)-g(n)<1-\delta$. Since $g(n+1)-g(n)=g^{\prime}(\xi)$ for a $\xi \in(n, n+1)$, thus $g^{\prime}(\alpha)<1-\delta$ and we obtain a contradiction. The proof in the case if $g$ is bounded from below is analogous. The function $g$ is thus unbounded from above and from below and continuous thus $g(\mathbb{R})=\mathbb{R}$. From here for every $x \in \mathbb{R}$ there exists a $y \in \mathbb{R}$ such that $g(y)=x$ and we have $|g(x)-x|=|g(g(y))-g(y)| \leq \delta$. Our system is thus restrictely b-stable. It is stable too since for every $\epsilon>0$ the positive number $\delta<1$ is good for the stability.

We prove that the maximal measure of stability does not exists for system (18). For every measure of stability $\Phi$ of this system we have $\Phi(1)<1$. Indeed, 
if $\Phi(1)=a \geq 1$, then for every function $g$ such that $|g(g(x))-g(x)| \leq a$ and $\left|g^{\prime}(x)-1\right| \leq a$ there exists a solution $f$ of the system such that $|g(x)-f(x)| \leq$ 1. This is impossible for the function $g(x)=0$ since $f(x)=x$ is the only solution of the system. The function $\Phi_{1}$ such that $\Phi_{1}(\epsilon)=\Phi(\epsilon)$ for positive $\epsilon \neq 1$ and $\Phi_{1}(1)=[\Phi(1)+1] / 2$ is the measure of stability of our system (see the end of the previous section) and $\Phi(1)<\Phi_{1}(1)$.

System (18) is equivalent to the equation

$$
|f(f(x))-f(x)|+\left|f^{\prime}(x)-1\right|=0 .
$$

Moreover, the stability and the b-stability for equation 19 is the same as for the system (18) since the inequality

$$
|f(f(x))-f(x)|+\left|f^{\prime}(x)-1\right| \leq \delta
$$

implies

$$
|f(f(x))-f(x)| \leq \delta \quad \text { and } \quad\left|f^{\prime}(x)-1\right| \leq \delta
$$

for every $\delta>0$.

Acknowledgement. I wish to thank the referee for a number of valuable remarks.

\section{References}

[1] Bahyrycz A., Forti's example on an unstable homomorphism equation, Aequationes Math. 74 (2007), 310-313.

[2] Baker J.A., Lawrence J., Zorzitto F., The stability of the equation $f(x+y)=f(x) f(y)$, Proc. Amer. Math. Soc. 74 (1979), 242-246.

[3] Baker J.A., The stability of the cosine equation, Proc. Amer. Math. Soc. 80 (1980), 411-416.

[4] Batko B., Stability of Dhombres' equation, Bull. Austral. Math. Soc. 70 (2004), 499505.

[5] Cholewa P.W., The stability of the sine equation, Proc. Amer. Math. Soc. 88 (1983), 631-634.

[6] Cholewa P.W., Remarks on the stability of functional equations, Aequationes Math. 27 (1984), 76-86.

[7] Chudziak J., Approximate dynamical systems on interval, Appl. Math. Lett. 25 (2012), no. 3, 352-357.

[8] Forti G.L., The stability of homomorphisms and amenability, with applications to functional equations, Abh. Math. Sem. Univ. Hamburg 57 (1987), 215-226. 
[9] Gavruta P., On the stability of some functional equations, in: Stability of mappings of Hyers-Ulam type, Hadronic Press Collection of Original Articles, Hadronic Press, Palm Harbor, Fla, USA, 1994, pp. 93-98.

[10] Gronau D., 21 Problem, Aequationes Math. 39 (1990), 311-312.

[11] Jabotinsky E., Analitic iteration, Trans. Amer. Math. Soc. 118 (1963), 457-477.

[12] Mach A., Moszner Z., On the stability of the translation equation in some classes functions, Aequationes Math. 72 (2006), 191-197.

[13] Moszner Z., The translation equation and its application, Demonstratio Math. 6 (1973), 309-327.

[14] Moszner Z., Structure de l'automate plein, réduit et inversible, Aequationes Math. 9 (1973), 46-59.

[15] Moszner Z., Les équations et les inégalités liées á l'équation de translation, Opuscula Math. 19 (1999), 19-43.

[16] Moszner Z., On the stability of functional equations, Aequationes Math. 77 (2009), 33-88.

[17] Moszner Z., On stability of some functional equations and topology of their target spaces, Ann. Univ. Paedagog. Crac. Stud. Math. 11 (2012), 69-94.

[18] Moszner Z., On the stability of the squares of some functional equations, Ann. Univ. Paedagog. Crac. Stud. Math. 14 (2015), 81-104.

[19] Moszner Z., Przebieracz B., Is the dynamical system stable?, Aequationes Math. 89 (2015), 279-296.

[20] Nikodem K., The stability of the Pexider equation, Ann. Math. Sil. 5 (1991), 91-93.

[21] Przebieracz B., On the stability of the translation equation, Publ. Math. 75 (2009), no. 1-2, 285-298.

[22] Przebieracz B., On the stability of the translation equation and dynamical systems, Nonlinear Anal. 75 (2012), no. 4, 1980-1988.

[23] Przebieracz B., Dynamical systems and their stability, Ann. Math. Sil. 28 (2014), 107109.

[24] Sibirsky S., Introduction to topological dynamics, Noordhoff International Publishing, Leiden, 1975.

\author{
Institute of Mathematics \\ Pedagogical University \\ PODCHORĄŻYCH 2 \\ 30-084 KRAKÓW \\ POLAND \\ e-mail: zmoszner@up.krakow.pl
}

